\title{
Synthesis and Characterization of Nanowires
}

\author{
R.G. Musket, T. Felter, A. Quong
}

\section{March 1, 2000}

U.S. Department of Energy

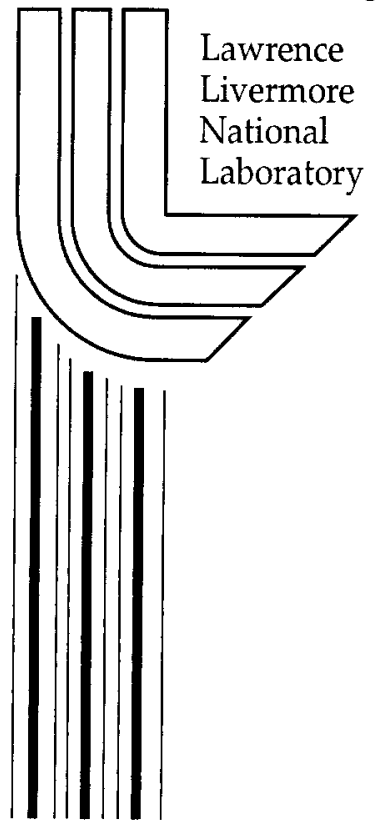

\section{PATENT CAUTION}

This document is transmitted prior to patent clearance and is made available in confidence to the addressee only. Its content is not to published, disclosed to others, or used for any other purpose until further notice from the Technical Information Department, Lawrence Livermore National Laboratory 


\section{DISCLAIMER}

This document was prepared as an account of work sponsored by an agency of the United States Government. Neither the United States Government nor the University of California nor any of their employees, makes any warranty, express or implied, or assumes any legal liability or responsibility for the accuracy, completeness, or usefulness of any information, apparatus, product, or process disclosed, or represents that its use would not infringe privately owned rights. Reference herein to any specific commercial product, process, or service by trade name, trademark, manufacturer, or otherwise, does not necessarily constitute or imply its endorsement, recommendation, or favoring by the United States Government or the University of California. The views and opinions of authors expressed herein do not necessarily state or reflect those of the United States Government or the University of California, and shall not be used for advertising or product endorsement purposes.

This work was performed under the auspices of the U. S. Department of Energy by the University of California, Lawrence Livermore National Laboratory under Contract No. W-7405-Eng-48. 
Laboratory Directed Research and Development Program

at

Lawrence Livermore National Laboratory

March 1, 2000

Final Report

SYNTHESIS AND CHARACTERIZATION OF NANOWIRES

(Tracking Code: 99-LW-026)

by

Principal Investigator:

Ronald G. Musket

Chemistry and Materials Science

Materials Science and Technology Division

LLNL, L-356

(925) 422-0483

Co-investigators: $\quad$ Thomas Felter

Andrew Quong

Chemistry and Materials Science

LLNL

Responsible A.D.: $\quad$ Harold Graboske

Chemistry and Materials Science

LLNL, L-90

(925) 422-7264

\begin{abstract}
With the dimensions of components in micro-electronic circuits shrinking, the phenomena associated with electronic conduction through wires and with device operation can be expected to change. For example, as the length of electrical conductors is reduced, ballistic transport will become the main mode of conduction. Sufficient reduction in the crosssectional area of conductors can lead to quantum confinement effects. Prior knowledge of the phenomena associated with decreasing size should help guide the designers of future, smaller devices in terms of geometry and materials. However, prior knowledge requires the availability of sufficiently small nanowires for experiments. To date, the smallest nanowires that have been fabricated and investigated had diameters of $8 \mathrm{~nm}$. We propose to extend the investigation of these size-related phenomena by synthesizing, using a novel version of nuclear, or ion, track lithography and characterizing, physically and electrically, nanowires with diameters $D$ of 1 to $5 \mathrm{~nm}$ and lengths $L$ of 2 to $250 \mathrm{~nm}$. Thus, by varying the dimensions of the nanowires, we will be able to determine experimentally when the ideas of macroscopic conductance break down and the conductance becomes dominated by quantum and ballistic effects. In our approach the nature of the small-diameter nanostructure formed can be controlled: Nanowires are formed when $L / D$ is large, and quantum dots are formed when both $\mathrm{L}$ and $\mathrm{D}$ are small. Theoretical calculations will be performed to both guide and understand the experimental studies. We have examined several aspects of this challenging problem and generated some promising results, but the project was not extended for the second year as planned. Thus, we did not have sufficient resources to complete the proof of concept.
\end{abstract}




\section{INTRODUCTION}

There has been considerable interest recently in fabricating and studying nanostructures and nanowires [1-3]. This has occurred because confinement of electrons to one dimension (quantum wire) has been predicted to lead to new electrical and optical properties [1]. With the lateral dimension, or diameter, of a semiconducting wire less than about $10 \mathrm{~nm}$, the energy separation between the quantized states will be greater than room-temperature thermal energies and technologically useful quantum wires will result [1]. For dimensions smaller than the characteristic scattering lengths, electron transport can be ballistic and highly sensitive to impurities and boundary conditions (e.g., contacts, surfaces, and interfaces) and simple one-electron theory no longer describes these effects [2]. When the diameter of a nanowire is comparable to the Fermi wavelength (about $1 \mathrm{~nm}$ in a metal and about $10-20 \mathrm{~nm}$ for semiconductors), quantized conductance occurs [4]. As a nanowire is stretched, the conductance decreases in a step-wise manner due the narrowing of the wire leads to sequential closing of quantized conductance channels [5]. This effect has led to a number of conductance studies of the uncharacterized, transient structures formed as scanning tunneling microscopy (STM) tips were retracted from liquid metal films [3].

The fabrication of 10-nm scale nanowires continues to be a major technological challenge with presently available lithography techniques $[1,2]$. Creation of such nanowires with large length-to-diameter ratios has previously been accomplished mostly by using electronbeam lithography, which has an ultimate resolution (i.e., wire diameter) of 10-20 nm [2]. The smallest nanowires reported to date were 8-nm gold wires [6] produced using "standard" nuclear track lithography [7], which consisted of filling the etched track in mica by electro-deposition of gold. We have pursued a novel version of nuclear, or ion, track lithography that permits fabrication of even smaller diameter nanowires.

Because there are both scientific and technological reasons for studying small, wellcharacterized nanowires, we planned to synthesize and characterize, both physically and electrically, nanostructures with diameters $D$ of 1 to $5 \mathrm{~nm}$ and lengths $L$ of 2 to $250 \mathrm{~nm}$. Nanowires are formed when L/D is large, and quantum dots are formed when $D$ is small and $L / D$ is approximately one. Theoretical calculations were planned to both guide and explain the experimental studies. Although the science of this study is most closely related to synthesis and characterization, the ultimate impact would be related to future microelectronic devices, including quantum dots for creating high efficiency lasers [1].

\section{PLANNED INVESTIGATIONS}

We designed a coordinated theoretical and experimental study of the fabrication and characterization, physically and electrically, of nanostructures. Both metallic and semiconductor nanostructures were to be investigated. The first principles calculations of the electronic states and conductance were to consider ballistic and quantized conduction effects by modeling nanostructures corresponding to those actually fabricated.

\section{A. Fabrication of nanostructures}

We planned to fabricate the nanostructures using a novel four step process. The first step is the directed passage of an appropriate ion through a layer of trackable material to create a latent nuclear track. A latent track is a cylindrical zone surrounding the straight path of an energetic ion that deposited energy mainly via interactions with the electrons of the material. When the energy deposited exceeds a critical value each latent track consists of a continuous, cylindrical volume that has been modified leaving a lower density, chemically different material. The lower density results from the creation of vacancies, and the chemical modification results from the destruction of the pre-existing electronic bonding in 
the material. Typical latent track diameters range from 7 to $30 \mathrm{~nm}$, depending on the energy density that the ion deposited into electronic stopping near the ion path, which varies with the ion species, the ion energy, and the material.

Unlike previous nuclear track lithography, where the latent tracks were chemically etched and then filled with metal, our process does not require that the trackable material be a resist, because the latent track will not be etched. However, the trackable material must still be a dielectric to allow the formation of electrically isolated, electrically conducting nanowires. In our approach, the length of the nanostructures is controlled by the thickness of the tracked layer, and the areal density of structures is determined by the ion irradiation fluence $\left(10^{6}-10^{11} / \mathrm{cm}^{2}\right)$.

To form the wires, conducting elemental species are infiltrated into the latent tracks (i.e., diffuse into the pores or vacancies of the tracks). Either a single elemental species or a mixture of elemental species can be used depending on the desire to make elemental or alloy wires. Infiltration of conducting elements from a thin film on the sample surface into the latent track cylinder can be accomplished at a temperature, $T_{d}$, high enough to result in diffusion of the conducting atoms throughout the latent track volume. The thin film could be present at the sample surface as a solid, liquid, or as a continuously replenished, very thin layer (i.e., approximately monolayer thick) with atoms arriving at the surface at a rate comparable to them infiltrating into the latent track.

In general, the temperature $T_{d}$ must be low enough that the amount of annealing of the latent track is insignificant, but high enough that the infiltration can be completed in a reasonable time. However, partial annealing of the latent track prior to infiltration may reduce the void volume of the latent track and, hence, reduce the amount of conducting atoms that can be infiltrated and, consequently, the final wire diameter. We planned to examine both thermally activated diffusion and electric-field-enhanced diffusion (with and without thermal activation) to fill the latent track with the elements of interest. To ensure separate wire and host phases, the trackable material and the conducting atomic species must have an insignificant mutual solubility for each other at the temperatures of interest and must not form any intermediate phases with one another.

Once the infiltration is completed, thermally driven self-assembly of the conducting wire should occur when the temperature of the infiltrated, tracked material is heated to a temperature, $T_{a}$, sufficient to anneal, or recrystallize, the latent track and to drive the conducting elements out of solution along a cylindrical front converging toward the center of the track leading to the formation of the nanowire. These processes can be monitored by resistance measurements and/or optical spectroscopy. After removal of the residual coating of conducting species, some or all of the tracked material may be removed to leave partially or fully free-standing nanowires for physical characterization. In general, the nanowires produced by this method will be polycrystalline, but dimensional constraints of the small diameter may lead to monocrystalline wires, especially if the tracks are created in a monocrystalline material.

An example system would be tracking through 200 -nm thick, thermal $\mathrm{SiO}_{2}$ on silicon and infiltration with either $\mathrm{Au}, \mathrm{Ag}, \mathrm{Cu}, \mathrm{Pd}$, or $\mathrm{Ge}$. An estimate of the maximum diameter of the nanowire formed by such an infiltration process can be obtained by assuming that the latent track has a density that is about ten percent less than that of the initial $\mathrm{SiO}_{2}$ layer (this amount of density deficit has been reported for tracks in mica) and has a diameter of $10 \mathrm{~nm}$ (which is typical of tracks in quartz). For infiltration of $\mathrm{Au}$ into $\mathrm{SiO}_{2}$ with initial density of $2.2 \mathrm{~g} / \mathrm{cm}^{3}$ and a latent track density of $2.0 \mathrm{~g} / \mathrm{cm}^{3}$, the resulting thermally assembled Au wire would have a diameter of $3.35 \mathrm{~nm}$, assuming that the Au atoms filled all the vacancy space 
in the latent track and that all the infiltrated $\mathrm{Au}$ atoms participated in the formation of the wire.

\section{B. Physical Characterization of Nanostructures}

Rutherford backscattering (RBS) of relatively dense arrays of latent tracks infiltrated with metals (and of subsequent nanowires) provided the depth distribution of the metals in silica layers and substrates even in the presence of metallic over layers and allow non-destructive evaluation of the state of the process. For example, 10-nm diameter nanowires at a density of $1 \times 10^{11} / \mathrm{cm}^{2}$ is equivalent to a density of nearly $10 \%$ of that of the host material, but, because RBS from heavy atoms (such as gold) in a light substrate (such as silica) can be analyzed at levels far below $0.1 \%$, we can determine the degree and uniformity of infiltration with a depth resolution of $<10 \mathrm{~nm}$. Furthermore, since the number of latent tracks will be known accurately by dosimetry, RBS will give an excellent measure of average wire diameter as a function of depth, assuming bulk density of the infiltrating metal. A simple hot stage would permit in situ monitoring of the infiltration and annealing steps and facilitate optimization of the fabrication procedures.

After formation, individual nanowires were to be resolved and characterized by scanning electron microscopy (SEM) and transmission electron microscopy (TEM). Low-energy, high-resolution SEM provides details of microtopography, and TEM yields the atomic structure. Sputter removal of the metal overlayer provides a high contrast specimen for conventional SEM and, for even better observation, the host dielectric itself can be removed by selective etching leaving the wires as a "bed of nails" protruding from the substrate. Such microscopy will provide independent determination of the efficiency of converting latent tracks into nanowires. These SEM samples are also appropriate for simple cathodoluminescence measurements. Moreover, standard silicon wafer thinning makes TEM relatively easy for specimens made with silicon substrates. Since the track orientation is set by the angle of the incident ion, we will not be limited to axial views in TEM. Oblique incidence angles will permit excellent lateral views to determine smoothness and uniformity along the length.

\section{Theoretical Calculation of the Electronic States and Conduction Phenomena}

The groundwork for computing the electrical properties of nanowires has been established [8], but only approximate models for the electronic structure of the wire have been implemented. The needed quantities are the electronic band energies and wave functions for the nanowire. The challenge is finding a method that will represent both the complexities of the electronic structure and will allow for the inclusion of the confinement. Because the nanowires we propose to fabricate have dimensions of a few $\mathrm{nm}$, we can apply first-principles electronic structure based methods to determine the electronic levels, from which we can calculate the electrical conductivity. The method we planned to use is a highly optimized method based on density functional theory and has been used to study the energetics of many large systems. The method uses a compact basis set of plane waves and localized atomic-like orbitals. Because the basis set is so small, approximately 20 to 30 basis functions per atom, we can study very large systems appropriate to the nanowires grown in this project. We expected to be able to calculate the conductance for single crystal wires and for wires with defects (e.g., impurities). 


\section{Electrical Characterization of Nanostructures}

For the electric-field enhanced diffusion, we planned to monitor the infiltration into the latent tracks by recording the resistance of the array of infiltrated latent tracks. We expected that once the tracks were filled the resistance would decrease significantly. In addition, annealing to temperatures above $\mathrm{T}_{\mathrm{a}}$ to anneal the latent track and to form the nanowires should be detected by further reductions in the resistance of the array. Once we have defined the process(es) that lead to formation of arrays of nanowires, we planned to create $\sim 1 \mu \mathrm{m}^{2}$ electrical contact pads (by conventional lithography) on samples tracked to $10^{8}$ tracks $/ \mathrm{cm}^{2}$, or $1 \mathrm{track} / \mu \mathrm{m}^{2}$. Thus, each pad would most probably make contact to the end of one nanowire and could be used to make the measurements described below.

When the radius of the nanowires is approximately the size of the Fermi wave vector of the material, we expect to observe conductance quantization (CQ). That is, the conductance will appear in steps of $2 e^{2} / \mathrm{h}$. This has been observed in other metals, but in these other experiments the geometry of the nanowires was transitory and was not, and could not, be determined, and the CQ was observed by analyzing the data from hundreds or thousands of wires. In this work, we will measure the CQ directly by placing the wires in a magnetic field and measuring the conductance as a function of field strength. By varying the magnetic field strength, the electronic levels shift so different conductance channels open and close which leads to CQ.

For the larger nanowires, where we do not expect to observe CQ, it is important to study the role of size on the conductance. The conductance will correspond to the expected bulk values for the larger diameter nanowires. However, as the wires get smaller the role of confinement and quantum effects will change the conductance. Thus, by varying the radius of the wires we will be able to determine when the ideas of macroscopic conductance break down and the conductance becomes limited by quantum effects. Short nanowires will exhibit ballistic conduction, which is controlled by electron-wall scattering instead of the electron-electron and electron-phonon interactions that control conduction in bulk materials.

\section{E. Collaborators and Capabilities}

The committed team has the expertise and experience with a unique combination of (1) a working experimental capability for tracking materials and (2) a demonstrated ability for performing the theoretical calculations, including first principles calculations of the electronic states and conductance in nanostructures. In addition, at LLNL we have the infrastructure available for heating and annealing materials and for physical characterization of the nanowires. A no cost collaboration had been established with the NSF-funded Independent Research Group on Nanostructured Materials at Johns Hopkins University for the electrical (and magnetic) characterization of any nanowires produced. Professors Peter Searson, Chia-Ling Chien, and Daniel Reich of JHU have agreed to perform the electrical (and magnetic) characterizations. Thus, we were well-positioned to undertake this study and to succeed.

\section{F. Project Plan and Benefits}

Due to the ground-breaking nature of this work and to the maximum annual resources available, we believed that this project should be funded over a two year period. For the first year we had planned to concentrate on the fabrication and characterization of metallic nanowires and to begin fabrication of semiconducting nanowires. The theoretical effort was to be concentrated on an understanding of the electronic nature of the model nanowires to be formed, which would serve to guide the experimental work with regard to sizes and 
materials. In the second year, the experiments were to have been devoted mainly to fabrication and characterization of the semiconducting nanowires, and the theoretical calculations were to have emphasized the electrical properties of the various nanowires formed, including defects (e.g., impurities, grain boundaries) and three-dimensional effects.

When completed this project would have provided valuable knowledge to the designers of future, smaller devices in terms of geometry and materials. In addition, this project would have positioned LLNL for a leadership role in understanding the physics of these nanostructure devices. Finally, the patenting of processes would place LLNL in a strong position for interactions with various industries concerning fabrication and applications of nanostructures.

\section{PROJECT ACCOMPLISHMENTS AND RESULTS FOR FY99}

\section{A. General}

We performed a thorough literature search about the nature of latent nuclear tracks in materials and about the diffusion of metals in oxides, completed four different series of thermally activated infiltration experiments, and prepared for electric-field enhanced diffusion experiments.

For most of our experiments we investigated the diffusion of metal atoms ( $\mathrm{Au}, \mathrm{Ag}$, and $\mathrm{Pd}$ ) into tracked layers of thermal fused silica $\left(\mathrm{SiO}_{2}\right)$ on $\mathrm{Si}$. The silica layer thicknesses included were $20,100,200$, and $1100 \mathrm{~nm}$. Some thermally activated experiments were performed to study diffusion of the same metal atoms into thick fused silica and thick tracked quartz (i.e., crystalline $\mathrm{SiO}_{2}$ ) samples. All samples were carefully cleaned prior to tracking or coating. After ultra-sonic machining, the samples were cleaned using isopropanol and UV/ozone. We know that such cleaning, if done properly, provides atomically clean oxide surfaces [9]. Following cleaning, two different types of tracked samples were created: (1) samples tracked and then sputter coated with a metal (pre-tracked samples) and (2) samples coated with a metal and then tracked (post-tracked samples). Finally, the non-tracked controls were either entire samples (first series) or a protected half of each tracked sample (series 2 through 4 ). The metals were present as solid layers with various thickness $(0.5 \mathrm{~nm}$ to $60 \mathrm{~nm})$. Our calculations using the TRIM program showed that $15 \mathrm{MeV}$ Xe ions can create a latent nuclear track at least $1500 \mathrm{~nm}$ long in thermal fused silica; thus, we expected the irradiation to result in latent tracks throughout the thicknesses of the silica layers. The energy losses of the high-energy Xe ions in the metal layers are negligible. All tracking irradiations were done with either 15.2 or $17 \mathrm{MeV} \mathrm{Xe}$ ions to a fluence of about $1 \times 10^{11} \mathrm{Xe} / \mathrm{cm}^{2}$, as measured by our sensitive, four-cup dosimetry system.

\section{B. First Series of Experiments}

For the first series of experiments we investigated the diffusion of $\mathrm{Au}$ atoms from $60-\mathrm{nm}$ thick coatings into 1100 -nm thick thermal fused silica (on silicon). The 6-mm diameter disk samples were designed for clamping to a small heater for heating in a vacuum chamber where in-situ Rutherford backscattering (RBS) analysis could be performed after each heating step. The 4-mm diameter Au deposits were centered on the samples. Total number of samples in this series was eight.

Based on a literature result that $\mathrm{Au}$ diffuses very rapidly in radiation-damaged fused silica (diffusivity of $3 \times 10^{-11} \mathrm{~cm}^{2} / \mathrm{sec}<60 \mathrm{C}[10]$ ), we expected that only short heatings at temperatures greater than $100 \mathrm{C}$ would be required to cause detectable diffusion of $\mathrm{Au}$ into 
the latent tracks. Our calculations using the RUMP program showed that RBS would allow us to detect the diffusion of Au atoms into the latent nuclear tracks provided the vacancy concentration in the track was at least one per atomic layer, which is the minimum expected in latent tracks.

Samples of each type were heated in vacuum at temperatures from $50 \mathrm{C}$ to over $600 \mathrm{C}$ for times of 10 to 40 minutes, but RBS did not show any evidence of diffusion of $\mathrm{Au}$ atoms into the tracked or non-tracked $\mathrm{SiO}_{2}$ layers. Our initial RBS geometry was chosen to allow us to determine the Au concentration throughout the fused silica layer, because we anticipated that the diffusion of $\mathrm{Au}$ into the latent tracks would be quite rapid. This geometry limited our analysis to Au that had diffused to depths $>100 \mathrm{~nm}$, due to the interference of the RBS signal from the gold coating. In subsequent experiments the RBS geometry was changed to allow analysis for diffusion depths $<50 \mathrm{~nm}$. Finally, after annealing at temperatures $\geq 450 \mathrm{C}$ for 10 minutes, there was evidence (visual, RBS, optical, and SEM) of gold segregation into thicker deposits. This result defined an upper limit on the temperature to be used to diffuse gold atoms into the tracks in fused silica.

Although we could not find any evidence in the literature for diffusion of elements from a layer into latent tracks, the reviewed literature supports our original hypothesis that such diffusion should occur due to the large vacancy concentration found in latent tracks. Consequently, we have decided that our first experiments were flawed by one or more of the following: (a) the thermal fused silica surfaces were contaminated and the gold was not in direct contact with the silica, (b) the irradiations did not go as planned (e.g., wrong ion, low fluence, or poor uniformity), (c) the gold does not wet the silica surface, and (d) the anneal times were too short.

\section{Second Series of Experiments}

We designed a second series of experiments to eliminate the potential flaws listed above and to increase the data output (i.e., anneal samples in parallel instead of in series). In this series we used 25-nm thick layers of $\mathrm{Au}, \mathrm{Pd}$, or $\mathrm{Ag}$ on carefully cleaned and handled rectangular (i.e., $9 \mathrm{~mm} \times 16 \mathrm{~mm}$ ) samples of $1100 \mathrm{~nm} \mathrm{SiO}{ }_{2}$ on $\mathrm{Si}$. In addition to improving cleanliness, we performed post-tracking irradiations under well-controlled conditions that allowed us to verify the ion, energy, fluence, and uniformity). Only half of each coated sample was irradiated. From the literature, we concluded that both $\mathrm{Pd}$ and $\mathrm{Ag}$ will wet silica. The diffusion coefficients of the three metals in radiation-damaged, or otherwise defected, silica are much smaller than reported in reference 10 (e.g., $10^{-14} \mathrm{~cm}^{2}$ $/ \mathrm{sec}$ at $150 \mathrm{C}$ for $\mathrm{Au}$ in electron-irradiated fused silica [11]). Thus, we used temperatures of $220 \mathrm{C}$ to $390 \mathrm{C}$ for much longer times (e.g., 8 to 72 hours) for heatings in nitrogen gas at atmospheric pressure. We heated the $\mathrm{Au}-, \mathrm{Pd}-$, and $\mathrm{Ag}$-coated samples at the same time and did the RBS analysis on both the irradiated and non-irradiated halves of the samples. The total sample matrix was 24 .

The only apparent preferential diffusion of metal into the tracked half of samples was for Ag sample heated at $390 \mathrm{C}$ for 8 hours. RBS showed that either the Ag diffused faster into the tracks than into the non-tracked silica or that the vertical segregation of the $\mathrm{Ag}$ film on the tracked area was greater than that on the non-tracked area. RBS measurements after removal of the $\mathrm{Ag}$ films revealed that there was not any $\mathrm{Ag}$ diffusion into either the tracked or the non-tracked areas. 


\section{Theoretical Calculations}

Using density functional theory we calculated the density of states for Pd nanowires and found it to be quite different from that calculated for bulk Pd. Figure 1 shows a comparison of the density of states calculated using versions of density functional theory for bulk Pd and our calculation for "FCC" Pd nanowires.

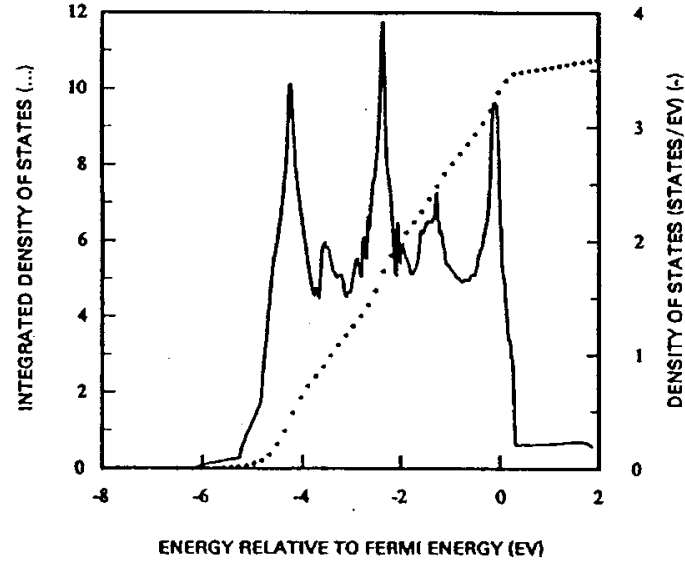

(a)

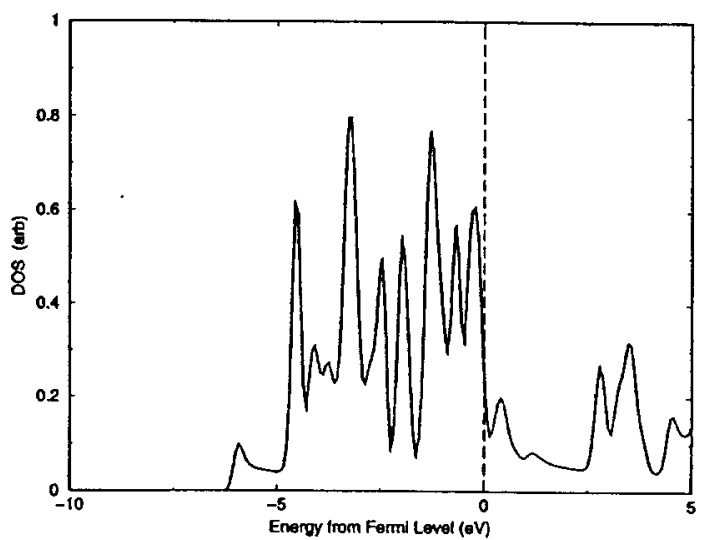

(b)

Figure 1: Calculated density of states for (a) bulk Pd (from Moruzzi et al, 1978) and (b) "FCC" Pd nanowire with $4 \mathrm{~A}$ atoms $+1 \mathrm{~B}$ atom $+4 \mathrm{~A}$ atoms $+1 \mathrm{~B}$ atom $+\ldots$.

Because the density of states for bulk and nanowire Pd are so different, the electrical properties will certainly be different. The calculations for the nanowires could be specialized to any nanowire we produced. Experimental evaluation of the electrical properties difference between bulk and nanowire of any fabricated nanowire was planned.

\section{E. Third Series of Experiments}

Continuing to use samples with the rectangular shape, we performed a third series of experiments on of post-irradiated samples using vacuum anneals to avoid oxidation of the $\mathrm{Ag}$ and $\mathrm{Pd}$ coatings. In addition, we limited the temperatures to low values (150 C and 225 C) to ensure that we were not annnealing out the latent tracks in the 20,100 , and $200 \mathrm{~nm}$ fused silica layers. There were several different sets of samples with the total number of samples being 88 :

(a) $0.5 \mathrm{~nm}$ and $25 \mathrm{~nm}$ thick layers $\mathrm{Au}, \mathrm{Ag}$, and $\mathrm{Pd}$ coatings on $200 \mathrm{~nm}$ thick $\mathrm{SiO}_{2}$ on $\mathrm{Si}$,

(b) $200 \mathrm{~nm}$ thick $\mathrm{SiO}_{2}$ on $\mathrm{Si}$ as witnesses of effects on non-coated samples, as potential samples to get Si to diffuse into tracks from the substrate, and as verification that tracking occurred by etching in HF acid,

(c) $0.5 \mathrm{~nm}$ and $25 \mathrm{~nm}$ thick layers of Au on $170 \mathrm{~nm}$ thick polycrystalline Si on $100 \mathrm{~nm}$ thick $\mathrm{SiO}_{2}$ on $\mathrm{Si}$, which were annealed in vacuum at $800 \mathrm{C}$ for 30 minutes to diffuse the $\mathrm{Au}$ to the polycrystalline $\mathrm{Si} / \mathrm{SiO}_{2}$ interface prior to tracking and lower temperature anneals, (d) $170 \mathrm{~nm}$ thick polycrystalline $\mathrm{Si}$ on $100 \mathrm{~nm}$ thick $\mathrm{SiO}_{2}$ on $\mathrm{Si}$ as witnesses of the noncoated samples and as potential samples to get $\mathrm{Si}$ to diffuse into tracks from the polycrystalline Si film, and

(e) Ge implanted into the Si substrate just below a thin $20 \mathrm{~nm}$ thick silica layer as potential samples to get $\mathrm{Ge}$ to diffuse into the tracks from the Si substrate, which were annealed at $1100 \mathrm{C}$ in vacuum for 3 hours to segregate the $\mathrm{Ge}$ at $\mathrm{SiO}_{2} / \mathrm{Si}$ interface prior to tracking and anneals at lower temperature. 
We performed RBS analysis of all samples at each stage of the processing, including removal of the metal coatings of samples that showed potential evidence of preferential diffusion into tracked areas. An example of the RBS spectra of such a case is shown in Figs. 2 and 3 , which provided the strongest evidence for such preferential diffusion. In Fig. 2(b), the simulated Au concentration of 67 at.ppm throughout the $\mathrm{SiO}_{2}$ layer (i.e., the flat part of the simulation between channels $\sim 610$ and $\sim 670$ ) is comparable to that expected for a successful preferential diffusion into tracks leading to $0.3 \mathrm{~nm}$ diameter wires. This demonstrates the high sensitivity of RBS for high-Z elements in a low-Z matrix and why RBS was the technique to assess initially the results of all of our diffusion experiments.

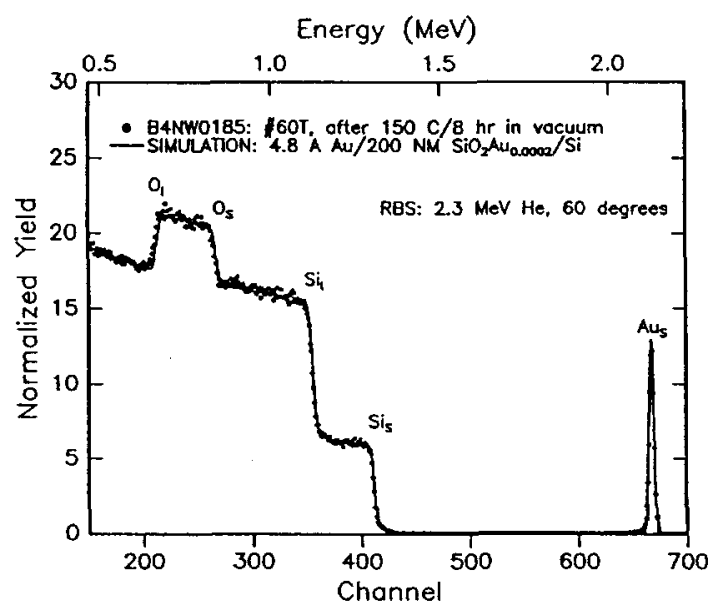

(a)

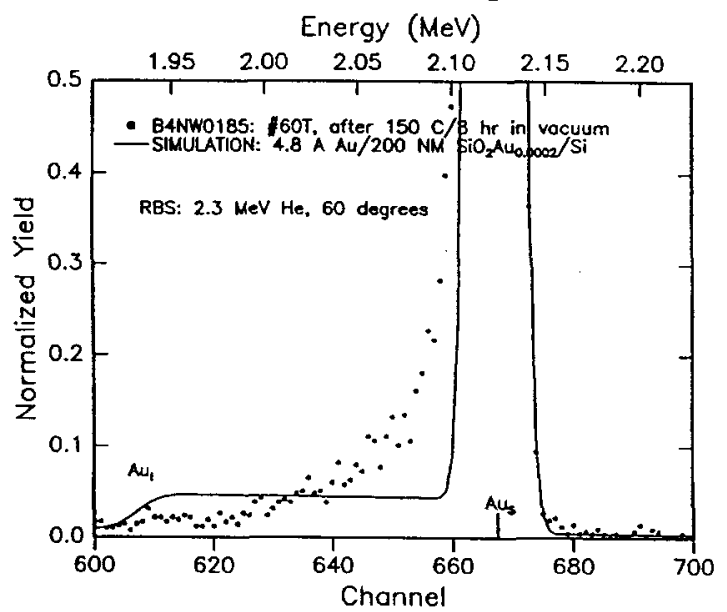

(b)

Figure 2: Comparison of RBS spectrum for the post-tracked side of a sample with $0.5 \mathrm{~nm}$ thick Au on $200 \mathrm{~nm}$ thick thermal fused silica on silicon after heating at $150 \mathrm{C}$ for 8 hours in vacuum. The simulation describes a structure assuming $0.48 \mathrm{~nm}$ thick Au on $200 \mathrm{~nm}$ thick $\mathrm{SiO}_{2}$ on $\mathrm{Si}$ with 67 at.ppm Au throughout the $\mathrm{SiO}_{2}$ layer. (a) Full spectrum and (b) Expanded spectrum showing only the region with the signal from Au with the plotted sensitivity increased by $60 \mathrm{X}$ compared to (a).

Because the Au coating was so thin we could see evidence for preferential diffusion into fused silica layer of the tracked half by directly comparing the Au signals (Fig. 3(a)) and subtracting the data for the non-tracked from the tracked (Fig. 3(b)); however, subsequent RBS after removal of the thin Au surface layer did not reveal any significant difference between the Au signals from the two halves of the sample. Thus, the apparent preferential diffusion must have been due to weak vertical segregation. 


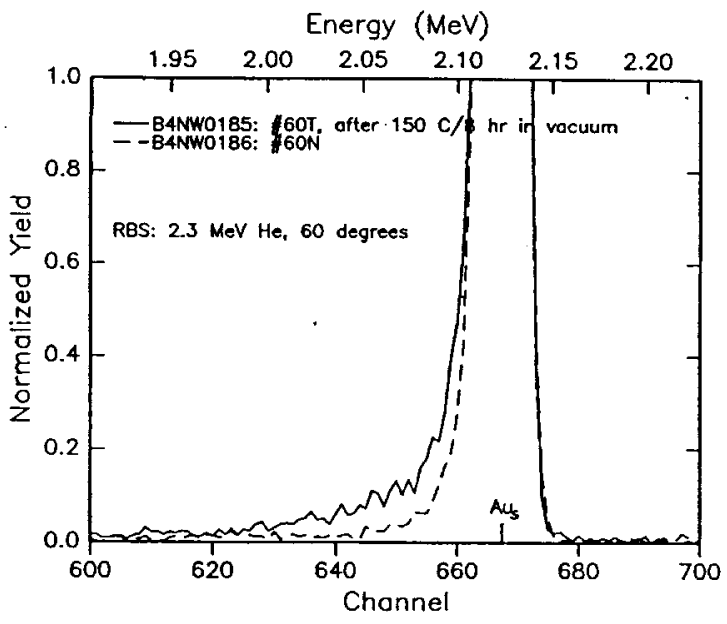

(a)

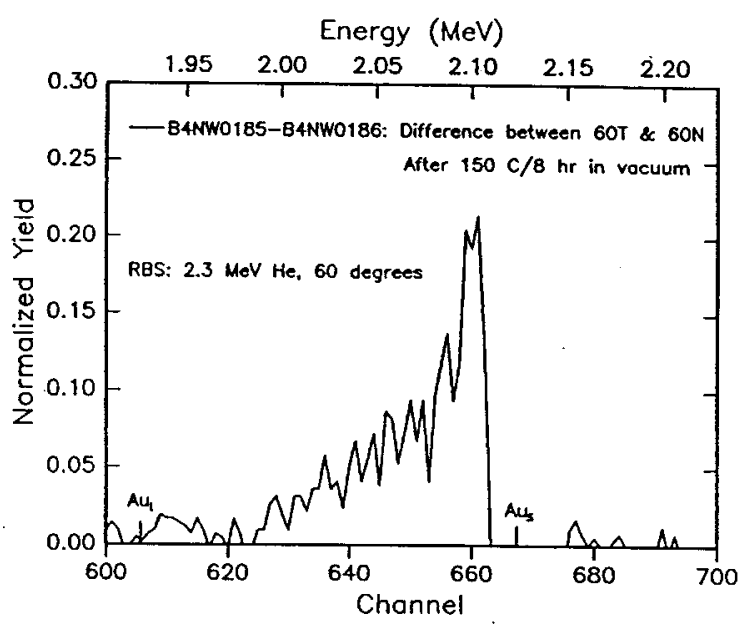

(b)

Figure 3: (a) Overlay of the as-annealed, Au RBS signals for tracked (T) and non-tracked (N) halves of the sample of Fig. 2 with $0.5 \mathrm{~nm}$ thick $\mathrm{Au}$ on $200 \mathrm{~nm}$ thick $\mathrm{SiO}_{2}$ on $\mathrm{Si}$ and (b) Au signal from the tracked half of the sample after subtracting the signal from the nontracked half.

SEM of the tracked halves of non-coated $\mathrm{SiO}_{2}$ on $\mathrm{Si}$ samples after etching in $\mathrm{HF}$ acid revealed a very rough surface due to the high density of tracks, whereas the non-tracked half did not have any significant roughness. Thus, we proved that latent tracks were being created, but that in the future we should do some irradiations at lower fluences to allow SEM observation of individual etched holes.

\section{F. Fourth Series of Experiments}

As in the previous series of experiments, we continued to use the rectangular samples and vacuum anneals to avoid oxidation of the $\mathrm{Ag}$ and $\mathrm{Pd}$ coatings. In the fourth series of experiments we examined the effects for pre-irradiated samples. We also added the following samples: thick fused silica, thick quartz, and $3500 \mathrm{~nm}$ thick mica mounted on $\mathrm{Si}$ substrates using carbon dag. As before, we limited the annealing temperatures for the fused silica samples to low values $(150 \mathrm{C}$ and $225 \mathrm{C})$ to ensure that we were not annnealing out the latent tracks. The quartz and mica samples were annealed at temperatures of 350-650 C and 350-550 C, respectively, because the literature indicated that track damage does not anneal in quartz at temperatures below $700 \mathrm{C}$ or in mica at temperatures below $600 \mathrm{C}$. There were several different sets of samples with the total number of samples being 104:

(a) $0.5 \mathrm{~nm}$ thick Au layers and $25 \mathrm{~nm}$ thick layers of $\mathrm{Au}, \mathrm{Ag}$, and Pd coatings on $200 \mathrm{~nm}$ thick $\mathrm{SiO}_{2}$ on $\mathrm{Si}$,

(b) $0.5 \mathrm{~nm}$ thick $\mathrm{Au}$ layers and $25 \mathrm{~nm}$ thick layers of $\mathrm{Au}, \mathrm{Ag}$, and Pd coatings on thick fused silica,

(c) $0.5 \mathrm{~nm}$ thick Au layers and $25 \mathrm{~nm}$ thick layers of $\mathrm{Au}, \mathrm{Ag}$, and Pd coatings on thick quartz,

(d) $0.5 \mathrm{~nm}$ thick Au layers and $25 \mathrm{~nm}$ thick layers of $\mathrm{Au}, \mathrm{Ag}$, and Pd coatings on mica mounted on $\mathrm{Si}$, and

(e) non-coated (i.e., silica layers on $\mathrm{Si}$ ) witnesses of irradiations to doses of $10^{9}, 10^{10}$, and $10^{11}$ tracks $/ \mathrm{cm}^{2}$ as samples for analysis of latent tracks by TEM, AFM, and positron annihilation and as verification that tracking occurred by etching in HF acid. 
As before, we performed RBS analysis of all coated samples at each stage of the processing, including after removal of the metal coatings from samples that had RBS results suggesting preferential diffusion into tracked areas. Although we did not find any convincing evidence of preferential diffusion into the tracked half of any of the thin or thick fused silica samples, we did verify that tracking is occurring by etching samples in HF acid (Fig. 4).
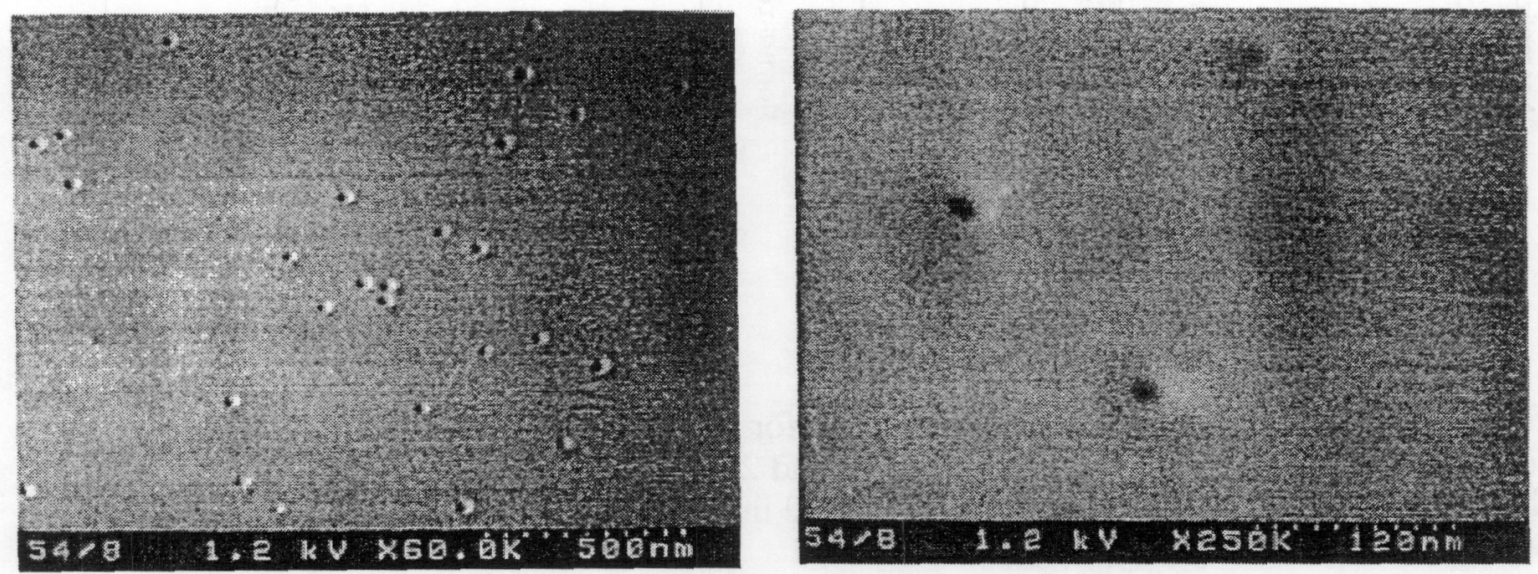

Figure 4: SEM of surface of $200 \mathrm{~nm}$ thick $\mathrm{SiO}_{2}$ layer on $\mathrm{Si}$ after tracking with $17 \mathrm{MeV} \mathrm{Xe}$ to a fluence of $10 \% / \mathrm{cm}^{2}$ and etching in $~ 8 \% \mathrm{HF}$ for 54 seconds at room temperature.

The holes in the surface of the oxide are the result of etching the damage in the tracks faster than etching the non-tracked materials surrounding each track. For the given measured fluence there should be $25 \pm 5$ tracks in the $2.5 \mu \mathrm{m}^{2}$ of the left micrograph and there are 28 . The etched holes have dark diameters of about $14 \mathrm{~nm}$, which is consistent with latent track diameters of $<10 \mathrm{~nm}$. Thus, the $17 \mathrm{MeV}$ Xe ions are creating latent tracks in the thermal fused silica layers.

Although comparison of the RBS spectra for the tracked area on the mica samples with the non-tracked areas suggested that preferential diffusion had occurred into the latent tracks, attempts to remove the metal layers from the surface of the mica were not successful. Either the mica was torn in the process or not enough of the metal could be removed uniformly.

The only confirmed indication that preferential diffusion occurred in the tracked region of a sample was found for a quartz sample that had been pre-irradiated, coated with $25 \mathrm{~nm}$ of $\mathrm{Au}$, and annealed in vacuum at $350 \mathrm{C}$ for 8 hours. As shown in Fig. 5(a), there is significantly more $\mathrm{Au}$ inside the tracked region than inside the non-tracked region. 


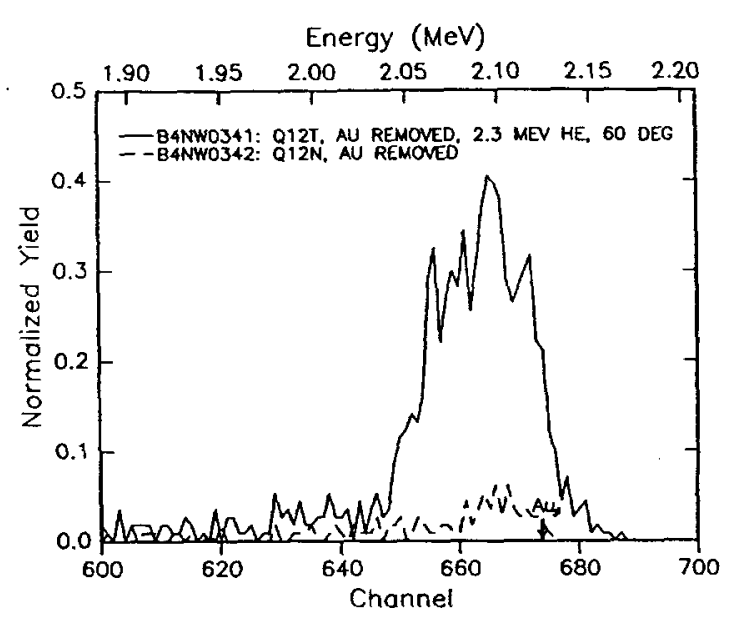

(a)

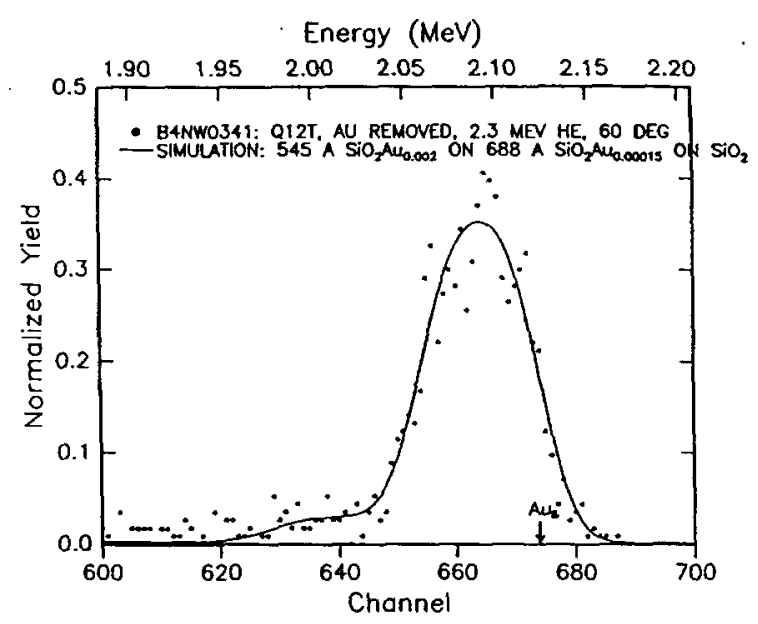

(b)

Figure 5: (a) Comparison of Au RBS signals for tracked (solid line) and non-tracked (dashed line) areas of sample that originally had $25 \mathrm{~nm}$ of $\mathrm{Au}$ on quartz after removal of the thick Au layer and (b) RUMP simulation (solid line) fitted approximately to the Au RBS signal (dots) for the tracked spectrum.

The ratio of $\mathrm{Au}$ in the tracked region to that in the non-tracked region is $A u_{\mathrm{T}} / \mathrm{Au}_{\mathrm{N}}>9$ with the total Au remaining in the tracked region being $\sim 3.2 \times 10^{14} \mathrm{Au} / \mathrm{cm}^{2}$. The approximate RUMP simulation for the first $54.5 \mathrm{~nm}$ of the $\mathrm{SiO}_{2}$ layer shows a Au concentration of 660 at.ppm, which is enough to form $\sim 1 \mathrm{~nm}$ diameter wires. The fitted Au concentration in the second part of the $\mathrm{SiO}_{2}$ layer (depth $54.5-123.3 \mathrm{~nm}$ ) is 50 at.ppm, which is enough to form $\sim 0.26 \mathrm{~nm}$ diameter wires. Very similar RBS results were found by comparing other spots in the tracked and non-tracked regions of this sample, confirming the conclusion stated above. Unfortunately, the FY99 budget was exhausted immediately after we obtained this very positive result, and the project was not funded for a second year, as originally proposed. Had additonal funds been available, we would have explored the effect of longer heating times followed by anneals at higher temperature to create nanowires imbedded in quartz. Because making electrical measurements on such nanowires would not be possible, we would have used SEM and TEM to characterize physically these nanowires. Thus, we would have proven the concept of producing nanowires by our process.

\section{G. Electric Field Enhanced Diffusion Experiments}

In preparation for performing electric field enhanced diffusion experiments, we modified the design of an existing vacuum hot stage that allows for heating up to eight samples at the same temperature while measuring the resistance of each sample. Sample temperatures of up to $340 \mathrm{C}$ in vacuum and in 1 atmosphere of nitrogen gas have been demonstrated, but we believe that temperatures near $500 \mathrm{C}$ would be achievable. We specialized the commercial program TestPoint to our needs for applying continuously the same voltage across all eight samples, sampling the resistance of each sample periodically, and saving the results to a file. However, our LDRD project ended before we could prepare the proper samples and pursue these experiments.

\section{CONCLUDING REMARKS}

We have explored a significant fraction of the parameter space related to the various processes available for creating $<5 \mathrm{~nm}$ nanowires using our novel version of ion track 
lithography. We have mostly learned what does not work, but we did obtain our most promising result at the end of FY99 (Fig. 5) for preferential diffusion of Au into tracked quartz relative to non-tracked quartz. This significant result was confirmed by RBS measurements at distinct regions of the same sample. However, we did not have the resources to reproduce this result on other samples and to determine the optimal annealing conditions to form Au nanowires. SEM and TEM would have been used to characterize the nanowires.

Over the course of our studies we concluded that the key consideration is the selection of the ideal trackable material. As given in our patent application related to these studies [12], we have developed the following criteria:

(a) Density of latent track must be less than that of the initial trackable material,

(b) Must be able to deposit or grow trackable material as a thin film to allow for precise control of the length of the nanowires,

(c) Annealing or recrystallization temperature must be considerably higher than the temperatures required for infiltration (crystalline materials probably preferred),

(d) Trackable material must be a dielectric material to allow for electric field enhanced diffusion of nanowire material into latent track and for measurement of electrical properties of the nanowires.

We believe that the following are examples of materials that fulfill all or some of the above criteria: quartz, fused silica, strontium titanate, Kapton, mica, polyethylene, and polycarbonate.

The difficulties we experienced in achieving success in this project within the one year of funding is consistent with the nature of the goal of unprecedented fabrication of $<5 \mathrm{~nm}$ diameter nanowires. From our review of the relevant literature and knowledge gained in this project, we remain convinced that our original hypothesis is correct and that preferential diffusion into latent tracks should occur due to the large vacancy concentration found in latent tracks and that nanowires can be fabricated and characterized as proposed. We will pursue resources to continue these studies in the future.

It is a pleasure to acknowledge the technical assistance of Phil Ramsey for the deposition of the coatings, Mervin Lawrence for the irradiations and some of the anneals, Larry Wagner for most of the anneals and modification of the hot stage, Jim Ferreira for the SEM analyses, and Ralph Pombo for setting up the TestPoint program for our field enhanced diffusion experiments. Work performed under the auspices of the U. S. Department of Energy by the Lawrence Livermore National Laboratory under contract W-7405-ENG-48. 


\section{REFERENCES}

1. M. Sundaram, S. A. Chalmers, P. F. Hopkins, and A. C. Gossard, Science 254, 1326 (1991).

2. D. K. Ferry and S. M. Goodnick, Transport in Nanostructures (Cambridge University Press, New York, 1997).

3. P. A. Serena and N. Garcia (eds), Nanowires (Kluwer Academic Publishers, Boston, 1997).

4. L. Olesen, K. Hansen, E. Laegsgaard, I. Stensgaard, and F. Besenbacher, ibid., p. 191-210.

5. W. A. DeHeer and D. Ugarte, ibid., p.227-236.

6. W. D. Williams and N. Giordano, Rev. Sci. Instrum. 55, 410 (1984) and Phys. Rev. B 33, 8146 (1986).

7. R. Spohr, Ion Tracks and Microtechnology, (Friedr. Vieweg \& Sohn Verlagsgesellschaft mbH, Braunschweig, 1990).

8. E. N. Bobachek, A. G. Scherbakov, and U. Landman, in P. A. Serena and N. Garcia (eds), Nanowires (Kluwer Academic Publishers, Boston, 1997)., p.35-60.

9. R. G. Musket, Appl. Surface Sci. 37, 55 (1989).

10. S. E. Ermatov, K. Togzhigitov, and V. A. Tskhai, Inorganic Materials 16, 1344 (1981).

11. V. V. Bolotov, V. M. Emeksuzyan, V. N. Spiridonov, K. Schmalz, and M. Trapp, Phys. Stat. Sol. A 113, 315 (1989).

12. R. G. Musket and T. E. Felter, "Formation of Nanometer-size Wires using Infiltration into Latent Nuclear Tracks," LLNL File No. IL-102283, patent applied for in November, 1999. 


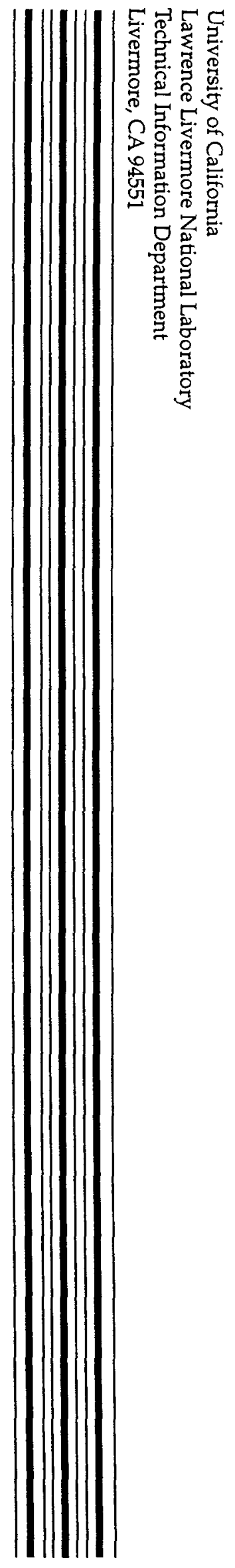

